\title{
Particle emission and gravitational radiation from cosmic strings: Observational constraints
}

\author{
Pierre Auclair@, ${ }^{1, *}$ Danièle A. Steer $\oplus^{1, \dagger}$ and Tanmay Vachaspati $\circledast^{2, \hbar}$ \\ ${ }^{1}$ Université de Paris, CNRS, Astroparticule et Cosmologie, F-75013 Paris, France \\ ${ }^{2}$ Physics Department, Arizona State University, Tempe, Arizona 85287, USA
}

(Received 4 December 2019; accepted 20 March 2020; published 7 April 2020)

\begin{abstract}
We account for particle emission and gravitational radiation from cosmic string loops to determine their effect on the loop distribution and observational signatures of strings. The effect of particle emission is that the number density of loops no longer scales. This results in a high-frequency cutoff on the stochastic gravitational wave background, but we show that the expected cutoff is outside the range of current and planned detectors. Particle emission from string loops also produces a diffuse gamma-ray background that is sensitive to the presence of kinks and cusps on the loops. However, both for kinks and cusps, and with mild assumptions about particle physics interactions, current diffuse gamma-ray background observations do not constrain $G \mu$.
\end{abstract}

DOI: 10.1103/PhysRevD.101.083511

\section{INTRODUCTION}

Most often the dynamics of local cosmic strings formed in a phase transition in the early Universe (see Refs. [1-3] for reviews) is described by the Nambu-Goto (NG) action. This approximation is valid when the microscopic width of the string

$$
w \sim \mu^{-1 / 2} \sim 1 / \eta
$$

(with $\mu$ the string tension and $\eta$ the energy scale of the phase transition) is very small relative to its characteristic macroscopic size $\ell-$ a situation which is well satisfied in the early Universe. Closed loops of NG strings lose energy slowly by radiating gravitational waves, and as a result, NG string networks contain numerous loops whose decay generate a stochastic gravitational wave background (SGWB) ranging over a wide range of frequencies [1]. Depending on the details of the particular cosmic string model, the corresponding constraints on the dimensionless string tension $G \mu$ from the SGWB are $G \mu \lesssim 10^{-7}$ at LIGO-Virgo frequencies [4] and $G \mu \lesssim 10^{-11}$ at Pulsar frequencies [5], whereas at LISA frequencies, one expects to reach $G \mu \lesssim 10^{-17}$ [6].

\footnotetext{
*auclair@apc.univ-paris7.fr

steer@apc.univ-paris7.fr

tvachasp@asu.edu
}

Published by the American Physical Society under the terms of the Creative Commons Attribution 4.0 International license. Further distribution of this work must maintain attribution to the author(s) and the published article's title, journal citation, and DOI. Funded by SCOAP ${ }^{3}$.
On the other hand, at a more fundamental level, cosmic strings are topological solutions of field theories. Their dynamics can therefore also be studied by solving the fieldtheory equations of motions. In studies of large-scale fieldtheory string networks [7-10], loops are observed to decay directly into particles and gauge boson radiation on a short timescale of order of the loop length. Hence, field-theory string network simulations predict very different observational consequences-in particular, no SGWB from loops.

Since field-theory and Nambu-Goto strings in principle describe the same physics, and hence lead to the same observational consequences, this is an unhappy situation. Based on high-resolution field-theory simulations, a possible answer to this long-standing conundrum was proposed in Ref. [11]. In particular, for a loop of length $\ell$ containing kinks, a new characteristic length scale $\ell_{0}=\ell_{\mathrm{k}}$ was identified, and it was shown that if $\ell \gtrsim \ell_{\mathrm{k}}$ gravitational wave emission is the dominant decay mode, whereas for smaller loops $\ell \lesssim \ell_{\mathrm{k}}$, particle radiation is the primary channel for energy loss. That is,

$$
\frac{d \ell}{d t}= \begin{cases}-\gamma_{\mathrm{d}}, & \ell \gg \ell_{\mathrm{k}} \\ -\gamma_{\mathrm{d}} \frac{\ell_{\mathrm{k}}}{\ell}, & \ell \ll \ell_{\mathrm{k}}\end{cases}
$$

where

$$
\gamma_{\mathrm{d}} \equiv \Gamma G \mu
$$

with $\Gamma \sim 50$ being the standard constant describing gravitational radiation from cosmic string loops [12-15]. Notice that Nambu-Goto strings correspond to $\ell_{\mathrm{k}} \rightarrow 0$, and if particle radiation is dominant for all loops, $\ell_{\mathrm{k}} \rightarrow \infty$. 
In practice, $\ell_{\mathrm{k}}$ is neither of these two limiting values, and in Ref. [11] was estimated (for a given class of loops with kinks) to be given by

$$
\ell_{\mathrm{k}} \sim \beta_{\mathrm{k}} \frac{w}{\Gamma G \mu},
$$

where $w$ is the width of the string, Eq. (1), and the constant $\beta_{\mathrm{k}} \sim \mathcal{O}(1)$. Note that the simulations of Ref. [11] consider the oscillations of loops with kinks over their entire lifetime. The results show episodic emission, with the net result of all the dynamics being the $1 / \ell$ behavior of Eq. (2).

If a loop contains cusps, then one expects the above to be modified to $[16,17]$

$$
\frac{d \ell}{d t}= \begin{cases}-\gamma_{\mathrm{d}}, & \ell \gg \ell_{\mathrm{c}} \\ -\gamma_{\mathrm{d}} \sqrt{\frac{\ell_{\mathrm{c}}}{\ell}}, & \ell \ll \ell_{\mathrm{c}},\end{cases}
$$

where

$$
\ell_{\mathrm{c}} \sim \beta_{\mathrm{c}} \frac{w}{(\Gamma G \mu)^{2}}
$$

with $\beta_{\mathrm{c}} \sim \mathcal{O}(1)$. We note that this $1 / \sqrt{\ell}$ dependence is less certain since, to the best of our knowledge, no field-theory simulations of loops with cusps over their entire lifetime exist. $^{1}$

The aim of this paper is to determine the observational effects-and corresponding constraints on $G \mu$ - of a finite, fixed, value of $\ell_{\mathrm{k}}$ or $\ell_{\mathrm{c}}$. A first immediate consequence of the presence of the fixed scale is that the distribution of loops $n(\ell, t)$, with $n(\ell, t) d \ell$ being the number density of loops with length between $\ell$ and $\ell+d \ell$ at time $t$, will no longer be scaling. That is, contrary to the situation for NG strings, the loop distribution will depend explicitly on $t$ as well as the dimensionless variable $\gamma=\ell / t$. We determine this nonscaling loop distribution $n(\gamma, t)$ in Sec. II, taking into account exactly (and for the first time) the backreaction of particle emission on the loop distribution.

We then study the consequence of the nonscaling distribution of non-self intersecting loops on the stochastic gravitational wave $(\mathrm{GW})$ background, determining the fraction of the critical density in GWs per logarithmic interval of frequency,

$$
\Omega_{\mathrm{gw}}\left(t_{0}, f\right)=\frac{8 \pi G}{3 \mathrm{H}_{0}^{2}} f \frac{\mathrm{d} \rho_{\mathrm{gw}}}{\mathrm{d} f}\left(t_{0}, f\right),
$$

\footnotetext{
${ }^{1}$ Reference [17] studies a single cusp on a field-theory string and gives the energy emitted per cusp, from which one deduces the $1 / \sqrt{\ell}$ behavior. It shows that the shape of the string is modified after the cusp event and argues that further smaller cusps will be formed in future loop oscillations. What we assume above is that the net result is a $1 / \sqrt{\ell}$ dependence.
}

where $\mathrm{H}_{0}$ is the Hubble parameter and the $d \rho_{g w} / d f$ factor is the energy density in gravitational waves per unit frequency $f$ observed today (at $t=t_{0}$ ). A scaling distribution of NG loops gives a spectrum which is flat at high frequencies [1]; we will show below that a consequence of the nonscaling of the loop distribution is the introduction of a characteristic frequency $f_{*}$, with $\Omega\left(f>f_{*}\right) \rightarrow 0$. The precise value of $f_{*}$ depends on $\ell_{\mathrm{k}}$ or $\ell_{\mathrm{c}}$, as well as $G \mu$. For cusps and kinks with $\ell_{\mathrm{c}}$ and $\ell_{\mathrm{k}}$ given, respectively, by Eqs. (2) and (4), the characteristic frequency $f_{*}$ is outside the LIGO and LISA band, provided $G \mu \gtrsim 10^{-17}$, and so in this case, the new cutoff will only be relevant for very light strings but for which the amplitude of the signal is below the observational thresholds of planned gravitational wave detectors.

In Sec. V, we turn to particle physics signatures. At lower string tensions $G \mu$, the gravitational signatures of strings weaken, while the particle physics ones are expected to increase. Following Ref. [18], we focus on so-called topdown models for production of ultrahigh-energy cosmic rays in which heavy particles, namely, the quanta of massive gauge and Higgs field of the underlying (local) field theory trapped inside the string, decay to give ultrahigh-energy protons and gamma rays. We focus on the diffuse gamma-ray flux which at $\mathrm{GeV}$ scales are constrained by Fermi-Lat [19]. However, taking into account backreaction of the emitted particles on the loop distribution, we find that current gamma-ray observations do not lead to significant constraints. (Early studies on the production of cosmic rays assumed NG strings and particle emission rates that were based on dynamics without taking backreaction into account. See Refs. [20-24] and [18] for a review. Other work has focused on strings with condensates, e.g., Refs. [25-27], or strings coupled to other fields such as Kaluza-Klein or dilaton fields $[28,29]$.)

This paper is organized as follows. In Sec. II, we determine the effect of an $\ell$-dependent energy loss,

$$
\frac{\mathrm{d} \ell}{\mathrm{d} t}=-\gamma_{\mathrm{d}} \mathcal{J}(\ell)
$$

on the loop distribution $n(\ell, t)$. The function $\mathcal{J}(\ell)$ will initially be left arbitrary. Specific cases corresponding to (i) NG loops with $\mathcal{J}=1$ (ii) loops with kinks [see Eq. (2)], and (iii) loops with cusps [see Eq. (4)] are studied in Secs. III A-III C. Given the loop distribution, we then use it to calculate the SGWB in Sec. IV and the predicted diffuse gamma-ray flux in Sec. V. We conclude in Sec. VI by discussing the resulting experimental constraints on $G \mu$.

\section{LOOP DISTRIBUTION}

All observational consequences of string loops depend on $n(t, \ell) \mathrm{d} \ell$, the number density of non-self intersecting loops with length between $\ell$ and $\ell+\mathrm{d} \ell$ at time $t$. In this section, we calculate $n(t, \ell)$ given (7); that is, we take into account the backreaction of the emitted particles on the 
loop distribution. As noted in the Introduction, the existence of the fixed scale $\ell_{\mathrm{k}}$ or $\ell_{\mathrm{c}}$ means that the loop distribution will no longer scale, that it will no longer be a function of the dimensionless variable $\gamma \equiv \ell / t$.

\section{A. Boltzmann equation and general solution}

The loop distribution satisfies a Boltzmann equation, which, taking into account the $\ell$ dependence of $\dot{\ell}$ (that is, the flux of loops in $\ell$ space), is given by [30]

$$
\left.\frac{\partial}{\partial t}\right|_{\ell}\left(a^{3} n(t, \ell)\right)+\left.\frac{\partial}{\partial \ell}\right|_{t}\left(\frac{\mathrm{d} \ell}{\mathrm{d} t} a^{3} n(t, \ell)\right)=a^{3} \mathcal{P},
$$

where $a(t)$ is the cosmic scale factor and the loop production function $\mathcal{P}(t, \ell)$ is the rate at which loops of length $\ell$ are formed at time $t$ by being chopped of the infinite string network. On substituting Eq. (7) into Eq. (8) and multiplying each side of the equation by $\mathcal{J}(\ell)$, one obtains

$$
\left.\frac{1}{\gamma_{\mathrm{d}}} \frac{\partial}{\partial t}\right|_{\ell} g(t, \ell)-\left.\mathcal{J}(\ell) \frac{\partial}{\partial \ell}\right|_{t} g(t, \ell)=a^{3} \mathcal{J}(\ell) \mathcal{P}(t, \ell),
$$

where

$$
g(t, \ell) \equiv \gamma_{\mathrm{d}} \mathcal{J}(\ell) a^{3}(t) n(t, \ell) .
$$

To solve (9), we first change variables from $(t, \ell)$ to

$$
\tau \equiv \gamma_{\mathrm{d}} t, \quad \xi \equiv \int \frac{\mathrm{d} \ell}{\mathcal{J}(\ell)} .
$$

Notice from (7) and (11) that for a loop formed at time $t_{i}$ with length $\ell_{i}$ its length at time $t$ satisfies

$$
\xi(\ell)+\gamma_{\mathrm{d}} t=\xi\left(\ell_{i}\right)+\gamma_{\mathrm{d}} t_{i} .
$$

In terms of these variables, Eq. (9) reduces to a wave equation with a source term

$$
\left.\frac{\partial}{\partial \tau}\right|_{\xi} g(\tau, \xi)-\left.\frac{\partial}{\partial \xi}\right|_{\tau} g(\tau, \xi)=\mathcal{S}(\tau, \xi),
$$

where

$$
\mathcal{S}(\tau, \xi)=a^{3}(\tau) \mathcal{J}(\xi) \mathcal{P}(\tau, \xi)
$$

We now introduce the light-cone variables

$$
2 u \equiv \tau-\xi, \quad 2 v \equiv \tau+\xi,
$$

so the evolution equation simply becomes

$$
\left.\frac{\partial}{\partial u}\right|_{v} g(u, v)=\mathcal{S}(u, v)
$$

which is straightforward to integrate. In the following, we neglect any initial loop distribution at initial time $t_{\text {ini }}$ (since this is rapidly diluted by the expansion of the Universe), so the general solution of (15), and hence the original Boltzmann equation (8), is

$$
g(u, v)=\int_{-v}^{u} \mathrm{~d} u^{\prime} S\left(u^{\prime}, v\right)
$$

Finally, one can convert back to the original variables $n(\ell, t)$ using (10) to find

$$
\begin{aligned}
n(t, \ell)= & \frac{1}{\gamma_{\mathrm{d}} \mathcal{J}(\ell) a^{3}(t)} \int_{-v(t, \ell)}^{u(t, \ell)} \mathrm{d} u^{\prime} a^{3}\left(u^{\prime}, v(t, \ell)\right) \\
& \times \mathcal{J}\left(u^{\prime}, v(t, \ell)\right) \mathcal{P}\left(u^{\prime}, v(t, \ell)\right)
\end{aligned}
$$

where $v(t, \ell)$ is obtained from Eqs. (11) and (14). Notice that $\mathcal{J}$ appears in two places: as an overall factor in the denominator as well as in the integrand.

\section{B. Solution for a $\delta$-function loop production function}

We now assume that all loops are chopped off the infinite string network with length $\alpha t$ at time $t$. This assumption, which has often been used in the literature, will lead to analytic expressions. The value $\alpha \sim 0.1$ is suggested by the NG simulations of Refs. [31,32], particularly in the radiation era. However, one should note that other simulations [33] are consistent with power-law loop productions functions [34,35], which have also been predicted analytically [36-38]. These will be considered elsewhere. Since $\alpha t \gg\left(\ell_{\mathrm{k}}, \ell_{\mathrm{c}}\right)$ for $\alpha \sim 0.1$, we expect that particle radiation from infinite strings will not affect the (horizon-size) production of loops from the scaling infinite string network, and hence we consider a loop production function of the form

$$
\mathcal{P}(t, \ell)=\mathrm{C} t^{-5} \delta\left(\frac{\ell}{t}-\alpha\right)
$$

where the constant $\mathrm{C}$, which takes different values in the radiation and matter eras, will be specified below. Substituting into (16), assuming $a \propto t^{\nu}$ (with $\nu=1 / 2$ in the radiation era and $\nu=2 / 3$ in the matter era) gives

$$
\begin{aligned}
g(u, v)= & \mathrm{C} \int_{-v}^{u} \mathrm{~d} u^{\prime} \mathcal{J}\left[\ell\left(u^{\prime}, v\right)\right] t\left(u^{\prime}, v\right)^{-5} a\left[t\left(u^{\prime}, v\right)\right]^{3} \\
& \times \delta\left[\frac{\ell\left(u^{\prime}, v\right)}{t\left(u^{\prime}, v\right)}-\alpha\right] .
\end{aligned}
$$

To evaluate this integral, in which $v=v(t, \ell)$ is fixed, let us denote the argument of the $\delta$ function by 


$$
y \equiv \frac{\ell\left(u^{\prime}, v\right)}{t\left(u^{\prime}, v\right)}-\alpha
$$

For the given $v$, the argument vanishes $(y=0)$ for some $u^{\prime}(v)$, which we will denote $u_{\star}(v)$ and which therefore satisfies

$$
\ell\left(u_{\star}, v\right)=\alpha t\left(u_{\star}, v\right) .
$$

Let us rewrite this more simply as $\ell_{\star}=\alpha t_{\star}$, where $\ell_{\star} \equiv$ $\ell\left(u_{\star}, v\right)=\ell_{\star}(v)$ and $t_{\star} \equiv t\left(u_{\star}, v\right)=t_{\star}(v)$. Now, from the $v$ equation in (14), one has $2 v=\gamma_{\mathrm{d}} t_{\star}(v)+\xi\left(\ell_{\star}(v)\right)$. Furthermore-since our final goal is to write the loop distribution in terms of $(t, \ell)$ (rather than $v$ )-we note from the same equation that $v$ is related to $(t, \ell)$ by $2 v=\gamma_{\mathrm{d}} t+\xi(\ell)$. Thus, $t_{\star}(t, \ell)$, which will be required below, is the solution of

$$
\gamma_{\mathrm{d}} t_{\star}+\xi\left(\alpha t_{\star}\right)=\gamma_{\mathrm{d}} t+\xi(\ell),
$$

which physically is simply relating the length of the loop $\alpha t_{\star}$ at its formation time $t_{\star}$, with its length $\ell$ at time $t$; see Eq. (12).

The final step needed to evaluate the integral in Eq. (II B) is the Jacobian of the transformation from $u^{\prime}$ to $y$, which, upon using (14), is given by

$$
\left.\frac{\partial}{\partial u^{\prime}}\right|_{v}\left(y\left(u^{\prime}, v\right)\right)=-\frac{\gamma_{\mathrm{d}} \mathcal{J}\left(\ell\left(u^{\prime}, v\right)\right) t\left(u^{\prime}, v\right)+\ell\left(u^{\prime}, v\right)}{\gamma_{\mathrm{d}} t\left(u^{\prime}, v\right)^{2}} .
$$

Evaluating this at $u^{\prime}=u_{\star}$ and using $\ell_{\star}=\alpha t_{\star}$ gives

$$
\left.\frac{\partial}{\partial u}\right|_{v}\left(y\left(u_{\star}, v\right)\right)=-\frac{\gamma_{\mathrm{d}} \mathcal{J}\left[\alpha t_{\star}(t, \ell)\right]+\alpha}{\gamma_{\mathrm{d}} t_{\star}(t, \ell)} .
$$

Having now expressed all the relevant quantities in terms of $(t, \ell)$, one can combine the above results and use the definition of $g$ in terms of $n(t, \ell)$ in Eq. (10) to find

$t^{4} n(t, \ell)=\mathrm{C} \frac{1}{\mathcal{J}(\ell)} \frac{\mathcal{J}\left(\alpha t_{\star}\right)}{\alpha+\gamma_{\mathrm{d}} \mathcal{J}\left(\alpha t_{\star}\right)}\left(\frac{t_{\star}}{t}\right)^{-4}\left(\frac{a\left(t_{\star}\right)}{a(t)}\right)^{3}$.

This equation, which is exact, is the central result of this section and gives the loop distribution for any form of energy loss $\mathrm{d} \ell / \mathrm{d} t=-\gamma_{\mathrm{d}} \mathcal{J}(\ell)$, provided the loop production function is a $\delta$ function. It generalizes and extends other approximate results which may be found in the literature.

For loops that are formed in a given era (either radiation or matter domination) and decay in the same era, the above solution reduces to

$$
t^{4} n(t, \ell)=\mathrm{C} \frac{1}{\mathcal{J}(\ell)} \frac{\mathcal{J}\left(\alpha t_{\star}\right)}{\alpha+\gamma_{\mathrm{d}} \mathcal{J}\left(\alpha t_{\star}\right)}\left(\frac{t_{\star}}{t}\right)^{3 \nu-4} .
$$

In the matter era, however, there also exists a population of loops which were formed in the radiation era, where $\mathrm{C}=\mathrm{C}_{\mathrm{R}}$, and decay in the matter era. Indeed, this population generally dominates over loops formed in the matter era. From (21), one can find a general expression for the distribution at any redshift $z$, provided the loops were formed in the radiation era $(\nu=1 / 2)$; it is given by

$$
\begin{aligned}
t^{4} n(t, \ell)= & \mathrm{C}_{\mathrm{R}} \frac{1}{\mathcal{J}(\ell)} \frac{\mathcal{J}\left(\alpha t_{\star}\right)}{\alpha+\gamma_{\mathrm{d}} \mathcal{J}\left(\alpha t_{\star}\right)}\left(\frac{t_{\star}}{t}\right)^{-5 / 2}(1+z(t))^{3} \\
& \times\left(2 \sqrt{\Omega_{\mathrm{R}}} \mathrm{H}_{0} t\right)^{3 / 2} .
\end{aligned}
$$

This reduces to (21) in the radiation era and has the correct scaling in the matter era.

In the following, we use standard Planck cosmology with Hubble constant $\mathrm{H}_{0}=100 h \mathrm{~km} / \mathrm{s} / \mathrm{Mpc}, h=0.678$, $\Omega_{M}=0.308, \Omega_{\mathrm{R}}=9.1476 \times 10^{-5}$, and $\Omega_{\Lambda}=1-\Omega_{M}-\Omega_{\mathrm{R}}$ [39]. We model the varying number of effective degrees of freedom in the radiation era through $H(z)=\mathrm{H}_{0} \mathcal{H}(z)$ with $\mathcal{H}(z)=\sqrt{\Omega_{\Lambda}+\Omega_{M}(1+z)^{3}+\Omega_{R} \mathcal{G}(z)(1+z)^{4}}$, where $\mathcal{G}(z)$ is directly related to the effective number of degrees of freedom $g_{*}(z)$ and the effective number of entropic degrees of freedom $g_{S}(z)$ by [40]

$$
\mathcal{G}(z)=\frac{g_{*}(z) g_{S}^{4 / 3}(0)}{g_{*}(0) g_{S}^{4 / 3}(z)} .
$$

We model this by a piecewise constant function whose value changes at the QCD phase transition $(T=200 \mathrm{MeV})$ and at electron-positron annihilation $(T=200 \mathrm{keV})$ :

$$
\mathcal{G}(z)= \begin{cases}1 & \text { for } z<10^{9} \\ 0.83 & \text { for } 10^{9}<z<2 \times 10^{12} \\ 0.39 & \text { for } z>2 \times 10^{12}\end{cases}
$$

\section{LOOP DISTRIBUTIONS FOR PARTICLE RADIATION FROM CUSPS AND KINKS}

Given a specific form of $\mathcal{J}(\ell)$, the loop distribution $n(\ell, t)$ is given by (21), where $t_{\star}(t, \ell)$ is obtained by solving (20). The existence or not of an analytical solution depends on the form of $\mathcal{J}(\ell)$. In this section, we consider three cases:

(1) Nambu-Goto loops.-Here, $\dot{\ell}=-\gamma_{\mathrm{d}}$ so that $\mathcal{J}=1$.

(2) Loops with kinks.-The asymptotic behavior of $\mathcal{J}(\ell)$ is given in Eq. (2). This can be captured, for instance, by $\mathcal{J}_{1}=1+\ell_{\mathrm{k}} / \ell$ or alternatively by

$$
\mathcal{J}_{\mathrm{k}}=\sqrt{1+\left(\frac{\ell_{\mathrm{k}}}{\ell}\right)^{2}} .
$$

This second form gives a simpler analytic expression for $t_{\star}$, and we work with it below. (We have checked 
that the differences in predictions arising from the choice of $\mathcal{J}_{1}$ or $\mathcal{J}_{\mathrm{k}}$ are negligible.)

(3) Loops with cusps.-Following Eq. (4), we take

$$
\mathcal{J}_{c}=\left[1+\left(\frac{\ell_{\mathrm{c}}}{\ell}\right)^{3 / 2}\right]^{1 / 3}
$$

which has the correct asymptotic behavior and also leads to analytical expressions. An alternative, and seemingly simpler, form $\mathcal{J}=1+\sqrt{\ell_{\mathrm{c}} / \ell}$ does not give analytical expressions for $n(t, \ell)$.

We now determine the corresponding loop distribution in scaling units, namely, in terms of the variables

$$
\gamma \equiv \frac{\ell}{t}, \quad \gamma_{\mathrm{k}}(t) \equiv \frac{\ell_{\mathrm{k}}}{t}, \quad \gamma_{\mathrm{c}}(t) \equiv \frac{\ell_{\mathrm{c}}}{t},
$$

and determine

$$
\mathcal{N}(t, \gamma) \equiv t^{4} n(t, \gamma)
$$

\section{A. NG strings}

A first check is that the above formalism yields the well-known, standard, loop distribution for NG strings $(\mathcal{J}=1)$. Equation (11) yields $\xi=\ell$, and from Eq. (20), it follows that

$$
\frac{t_{\star}}{t}=\frac{\gamma+\gamma_{\mathrm{d}}}{\alpha+\gamma_{\mathrm{d}}} .
$$

Hence, from Eq. (22),

$$
\mathcal{N}_{N G}(t, \gamma)=\mathrm{C} \frac{\left(\alpha+\gamma_{\mathrm{d}}\right)^{3(1-\nu)}}{\left(\gamma+\gamma_{\mathrm{d}}\right)^{4-3 \nu}}
$$

which is the standard scaling NG loop distribution for a delta-function loop production function [1]. In the radiation/matter eras, and on the scales $\alpha \gg \gamma_{\mathrm{d}}$ observed in simulations, comparison with the numerical results of Refs. [31-33] sets the values of $\mathrm{C}$ to, respectively,

$$
\begin{aligned}
& \mathrm{C}_{\mathrm{R}} \alpha^{3 / 2} \simeq 0.18 \quad\text { (radiation era }) \\
& \mathrm{C}_{\mathrm{M}} \alpha \simeq 0.27 \quad(\text { matter era })
\end{aligned}
$$

The scaling distribution Eq. (30) is shown in the black (solid) curve in Fig. 1, in which we have taken $\alpha=0.1$, $\gamma_{\mathrm{d}}=10^{-6}$, and $\nu=1 / 2$ (radiation era).

\section{B. Loops with kinks}

From Eq. (11), with $\mathcal{J}_{k}$ given Eq. (26), we now have $\xi(\ell)=\sqrt{\ell^{2}+\ell_{\mathrm{k}}^{2}}$. Thus, from Eq. (20), $t_{\star}$ satisfies a quadratic equation with solution

$$
\frac{t_{\star}}{t}=\frac{-\bar{\gamma}\left(\frac{\gamma_{\mathrm{d}}}{\alpha}\right)+\sqrt{\bar{\gamma}^{2}-\gamma_{\mathrm{k}}^{2}\left(1-\left(\frac{\gamma_{\mathrm{d}}}{\alpha}\right)^{2}\right)}}{\alpha\left(1-\left(\frac{\gamma_{\mathrm{d}}}{\alpha}\right)\right)},
$$

where $\gamma_{\mathrm{k}}(t)$ is given in (28) and

$$
\bar{\gamma}(t, \gamma) \equiv \gamma_{\mathrm{d}}+\sqrt{\gamma_{\mathrm{k}}^{2}(t)+\gamma^{2}}
$$

Since $\alpha \sim 0.1$ and $\gamma_{\mathrm{d}} \equiv \Gamma G \mu \lesssim 10^{-6}$ (from cosmic microwave background constraints on cosmic strings [41]), in

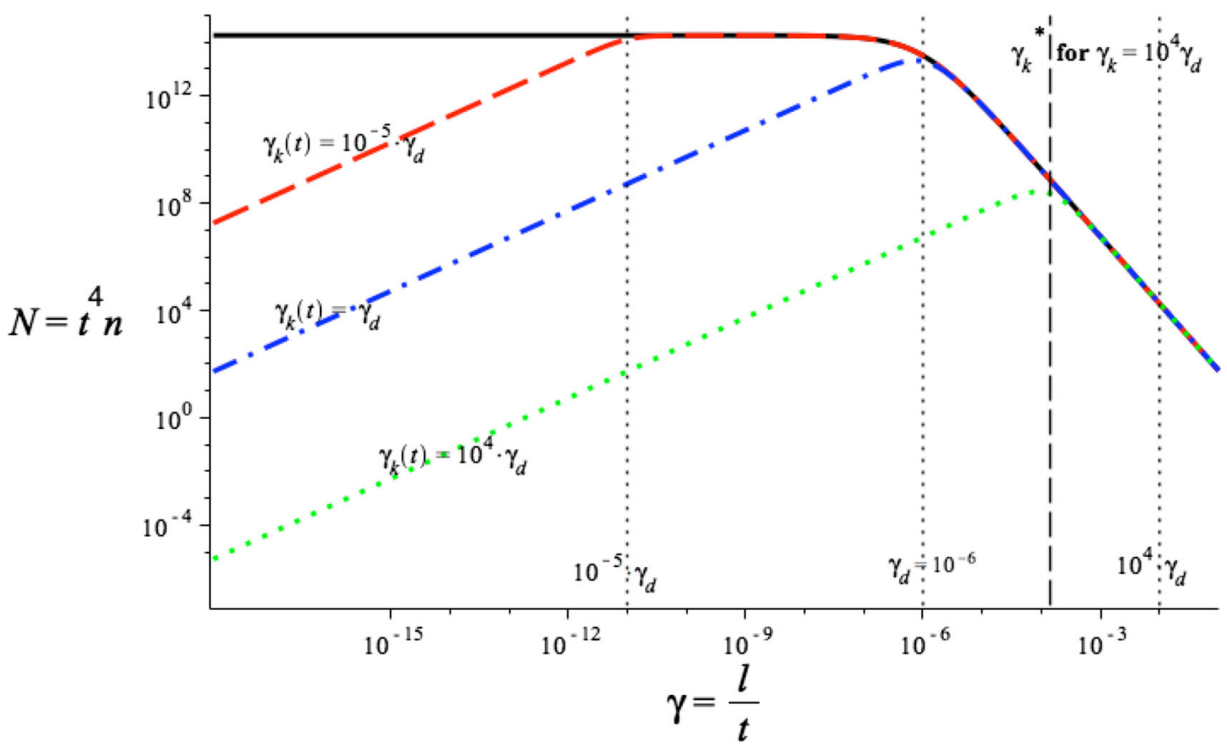

FIG. 1. Loop distribution for kinks in the radiation era, with $\alpha=0.1$ and $\gamma_{\mathrm{d}}=10^{-6}$, and at several different epochs. Black solid line: $\gamma_{\mathrm{k}}=0(t \rightarrow \infty)$, the NG loop distribution. Red dashed line: $\gamma_{\mathrm{k}}(t)=10^{-5} \gamma_{\mathrm{d}}$ (corresponding to $t=10^{5} t_{k}$ ). Blue dotted-dashed line: $\gamma_{\mathrm{k}}(t)=\gamma_{\mathrm{d}}$ (corresponding to $t=t_{k}$ ). Green dotted line: $\gamma_{\mathrm{k}}(t)=10^{4} \gamma_{\mathrm{d}}$ (corresponding $t=10^{-4} t_{k}$ ). 
our analytical expressions below we ignore terms in $\gamma_{\mathrm{d}} / \alpha$ so that $\left(\alpha t_{\star} / t\right)^{2}=\bar{\gamma}^{2}-\gamma_{\mathrm{k}}^{2}(t)$. (This approximation was not used in our numerical calculations.) Thus, from Eq. (21) we find, assuming $\alpha \gg \gamma_{\mathrm{d}}$,

$\mathcal{N}(t, \gamma)=\mathrm{C} \alpha^{3(1-\nu)}\left(\frac{\bar{\gamma}^{2}(t, \gamma)}{1+\gamma_{\mathrm{k}}^{2}(t) / \gamma^{2}}\right)^{1 / 2}\left(\bar{\gamma}^{2}(t, \gamma)-\gamma_{\mathrm{k}}^{2}(t)\right)^{\frac{3 \nu-5}{2}}$ where $\gamma \leq \alpha$.

This distribution, in the radiation era, is plotted in Fig. 1 for illustrative values of $\gamma_{\mathrm{k}}(t)$, with $\gamma_{\mathrm{d}}=10^{-6}, \alpha=0.1$.

The important qualitative and quantitative features to notice are the following:

(i) The existence of the fixed scale $\ell_{\mathrm{k}}$ gives rise to a nonscaling distribution: $\mathcal{N}$ is explicitly $t$ dependent.

(ii) When $\gamma_{\mathrm{k}} \rightarrow 0$, namely, when $t \rightarrow \infty$, Eq. (33) reduces to the standard scaling NG loop distribution given in Eq. (30) (in the limit $\alpha \gg \gamma_{d}$ ).

(iii) For $\gamma \gg \gamma_{\mathrm{k}}(t)$, the loop distribution is scaling since $\bar{\gamma} \sim \gamma+\gamma_{\mathrm{d}}$, so

$$
\mathcal{N}(t, \gamma) \simeq \mathrm{C} \alpha^{3(1-\nu)}\left(\gamma+\gamma_{\mathrm{d}}\right)^{3 \nu-4}
$$

This behavior is clear in Fig. 1, in which for $\gamma \gg \gamma_{\mathrm{k}}(t)$ the various curves coincide with the NG curve. Hence, for loops of these lengths, gravitational radiation is important, but particle radiation plays no role. Furthermore:

(a) when $\gamma_{\mathrm{d}} \gg \gamma \gg \gamma_{\mathrm{k}}$, the distribution is flat; see Fig. 1's dashed red curve.

(b) when $\gamma \gg\left(\gamma_{\mathrm{d}}, \gamma_{\mathrm{k}}\right), \mathcal{N}$ drops off as $\gamma^{3 \nu-4}$, as for NG loops, a dependence which is simply due to the expansion of the Universe.

(iv) For $\gamma \ll \gamma_{\mathrm{k}}(t)$, the distribution no longer scales because of particle radiation. Indeed, $\bar{\gamma} \sim \gamma_{\mathrm{k}}(t)+\gamma_{\mathrm{d}}$, so

$\mathcal{N} \simeq \mathrm{C} \alpha^{3(1-\nu)} \gamma_{\mathrm{d}}^{\frac{3 \nu-5}{2}}\left(\frac{\gamma}{\gamma_{\mathrm{k}}(t)}\right)\left(2 \gamma_{\mathrm{k}}(t)+\gamma_{\mathrm{d}}\right)^{\frac{3 \nu-5}{2}}\left(\gamma_{\mathrm{k}}(t)+\gamma_{\mathrm{d}}\right)$.

This linear dependence on $\gamma$ for $\gamma \ll \gamma_{k}$ is visible in Fig. 1. Notice that when $\gamma_{\mathrm{d}} \ll \gamma_{\mathrm{k}}$ there is no plateau in the distribution, which goes from the linear behavior (35) to the scaling behavior (34), at a value of $\gamma$ obtained by equating these two equations, namely,

$$
\gamma_{\mathrm{k}}^{*}(t) \simeq \sqrt{2 \gamma_{\mathrm{k}} \gamma_{\mathrm{d}}}
$$

This is clearly visible in the dotted green curve in Fig. 1.

When $\gamma_{\mathrm{k}}(t) \ll \gamma_{\mathrm{d}}$, an excellent approximation to the distribution is

$$
\mathcal{N}(\gamma, t) \simeq \mathrm{C} \alpha^{3(1-\nu)} \frac{1}{\mathcal{J}(\gamma, t)}\left(\gamma+\gamma_{\mathrm{d}}\right)^{3 \nu-4},
$$

where, for the kinks considered here,

$$
\mathcal{J}(\gamma, t)=\sqrt{1+\left(\frac{\gamma_{\mathrm{k}}(t)}{\gamma}\right)^{2}} .
$$

On the other hand, when $\gamma_{\mathrm{k}}(t) \geq \gamma_{\mathrm{d}}$, the distribution changes behavior, and for $\gamma_{\mathrm{k}}(t) \gg \gamma_{\mathrm{d}}$, its amplitude is significantly suppressed due to particle emission. Indeed, when $\gamma=\gamma_{\mathrm{k}}^{*}(t)$, which is at the maximum of $\mathcal{N}$ (see the green curve, Fig. 1), $\mathcal{N}$ scales as $\gamma_{\mathrm{k}}^{-(4-3 \nu) / 2}$, which decreases with increasing $\gamma_{\mathrm{k}}$. The equality $\gamma_{\mathrm{d}}=\gamma_{\mathrm{k}}(t)$ defines a characteristic time $t_{k}$ by

$$
t_{k} \equiv \frac{\ell_{\mathrm{k}}}{\gamma_{\mathrm{d}}} .
$$

For $t \ll t_{k}$, particle emission is dominant, $\gamma_{\mathrm{k}}(t) \geq \gamma_{\mathrm{d}}$, and the distribution is suppressed. Using $\ell_{\mathrm{k}}$ given by Eq. (3),

$$
t_{k}=\beta_{k} \frac{t_{\mathrm{pl}}}{\Gamma^{2}(G \mu)^{5 / 2}} \simeq \beta_{k} t_{\mathrm{eq}}\left(\frac{2.5 \times 10^{-24}}{G \mu}\right)^{5 / 2},
$$

or in terms of redshift,

$$
z_{k} \simeq z_{\mathrm{eq}}\left(\frac{G \mu}{2.5 \times 10^{-24}}\right)^{5 / 4} \frac{1}{\sqrt{\beta_{k}}}
$$

where $z_{\text {eq }} \simeq \Omega_{M} / \Omega_{\mathrm{R}} \sim 3367$. The left-hand (LH) panel of Fig. 2 shows the loop distribution for different redshifts for $\ell_{\mathrm{k}}$ given in Eq. (3) and $\beta_{k}=1$. The effect of the suppression of the loop distribution at $z \gg z_{k}$ on the SGWB will be discussed in Sec. IV.

\section{Loops with cusps}

For loops with cusps, where $\mathcal{J}=\mathcal{J}_{\text {c }}$ given in Eq. (27), the analysis is very similar. We only give the salient features. As for kinks (see Eq. (37), one can define a characteristic time through $\gamma_{\mathrm{d}}=\gamma_{\mathrm{c}}(t)$, namely,

$$
t_{c} \equiv \frac{\ell_{\mathrm{c}}}{\gamma_{\mathrm{d}}}
$$

and again, as for kinks, when $t \ll t_{c}$, the effects of particle radiation are more important, and the loop distribution is suppressed. For $\ell_{\mathrm{c}}$ given in Eq. (5), we have

$$
t_{c}=\beta_{c} \frac{t_{\mathrm{pl}}}{\Gamma^{3}(G \mu)^{7 / 2}} \simeq \beta_{c} t_{\mathrm{eq}}\left(\frac{4.6 \times 10^{-18}}{G \mu}\right)^{7 / 2},
$$

or in terms of redshift, 

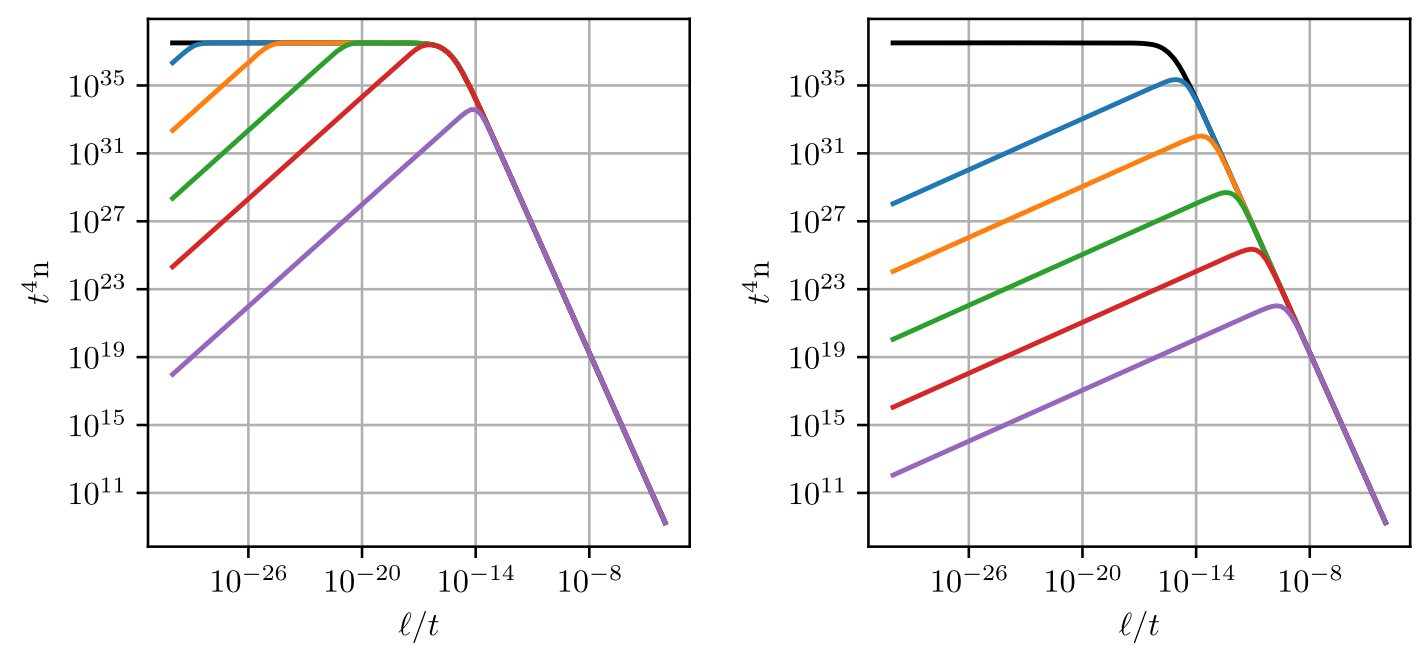

FIG. 2. Loop number density $\mathcal{N}=t^{4} n$ for kinks (left-hand panel) and cusps (right-hand panel), for $G \mu=10^{-17}$. Thus, $z_{k} \sim 10^{12}$ and $z_{c} \sim 10^{4}$. From bottom to top, the curves show snapshots of the loop distribution at redshifts $z=10^{13}, 10^{11}, 10^{9}, 10^{7}, 10^{5}$, and the black curve is the scaling loop distribution at $z \rightarrow 0$. The loop distributions are suppressed for $z \gg z_{k}$ or $z \gg z_{c}$.

$$
z_{c} \simeq z_{\mathrm{eq}}\left(\frac{G \mu}{4.6 \times 10^{-18}}\right)^{7 / 4} \frac{1}{\sqrt{\beta_{c}}}
$$

For the relevant range, namely, $G \mu<10^{-6}$, we have $z_{c}<z_{k}$, and hence the observational consequences of cusps, both on the SGWB and the diffuse gamma-ray background, are expected to be more significant than those of kinks - since, as discussed above, the loop distribution is suppressed when $z<\left(z_{c}, z_{k}\right)$; see Fig. 2.

The explicit $\gamma$ dependence of the distribution is the following. First, substituting $\mathcal{J}_{\mathrm{c}}$ in the definition of $\xi(\gamma)$ and $t_{*}$, Eqs. (11) and (20), respectively, we find

$$
\begin{aligned}
\xi(\ell) & =\left(\ell^{3 / 2}+\ell_{\mathrm{c}}^{3 / 2}\right)^{2 / 3}, \\
\left(\frac{\alpha t_{\star}}{t}\right)^{3 / 2} & =\left[\gamma_{\mathrm{d}}+\left(\gamma^{3 / 2}+\gamma_{\mathrm{c}}^{3 / 2}\right)^{2 / 3}\right]^{3 / 2}-\gamma_{\mathrm{c}}^{3 / 2} \text { for } \alpha \gg \gamma_{\mathrm{d}} .
\end{aligned}
$$

It then follows from Eq. (22) that the resulting distribution again scales for $\gamma \gg \gamma_{c}$, where it is given by Eq. (34); and for $\gamma \ll \gamma_{\mathrm{d}}, \mathcal{N} \propto \sqrt{\gamma}$. When $\gamma_{\mathrm{c}} \gg \gamma_{\mathrm{d}}$, we find

$$
\mathcal{N} \propto \begin{cases}\gamma^{3 \nu-4} & \left(\gamma \gg \gamma_{c}^{*}\right) \\ \sqrt{\gamma} & \left(\gamma \ll \gamma_{c}^{*}\right)\end{cases}
$$

where

$$
\gamma_{\mathrm{c}}^{*} \simeq\left(\gamma_{\mathrm{d}} \sqrt{\gamma_{\mathrm{c}}}\right)^{2 / 3}
$$

\section{STOCHASTIC GRAVITATIONAL WAVE BACKGROUND}

The stochastic GW background $\Omega_{\mathrm{gw}}\left(t_{0}, f\right)$ given in (6) is obtained by adding up the GW emission from all the loops throughout the whole history of the Universe which have contributed to frequency $f$. Following the approach developed in Refs. [1,15,42],

$$
\Omega_{\mathrm{gw}}(\ln f)=\frac{8 \pi G^{2} \mu^{2} f}{3 \mathrm{H}_{0}^{2}} \sum_{j=1}^{\infty} C_{j}(f) P_{j},
$$

where

$C_{j}(f)=\frac{2 j}{f^{2}} \int_{0}^{z_{\text {friction }}} \frac{d z}{H(z)(1+z)^{6}} n\left(\frac{2 j}{(1+z) f}, t(z)\right)$

and $z_{\text {friction }}$ is the redshift below which friction effects on the string dynamics become negligible [1],

$$
z_{\text {friction }} \simeq z_{\mathrm{eq}}\left(4.4 \times 10^{16}\right)\left(\frac{G \mu}{10^{-11}}\right) .
$$

The $C_{j}$ depend on the loop distribution $n(\ell, t)$ through $n(2 j /((1+z) f), t(z))$, while the $P_{j}$ are the "average loop gravitational wave power spectrum," namely, the power emitted in gravitational waves in the $j$ th harmonic of the loop. By definition of $\Gamma$, these must be normalized to

$$
\Gamma=\sum_{j=1}^{\infty} P_{j} .
$$

For loops with kinks, $P_{j} \propto j^{-5 / 3}$, whereas for loops with cusps, $P_{j} \propto j^{-4 / 3}[1,12,43]$.

As explained above, the effect of $\gamma_{\mathrm{k}}$ and $\gamma_{\mathrm{c}}$ on the loop distribution is particularly important at large redshifts $z>\left(z_{c}, z_{k}\right)$, and hence in the radiation era. Therefore, we expect the effect of particle radiation to be visible in the high-frequency part of the spectrum. This is indeed observed in Fig. 3, in which the LH panel is for kinks with 

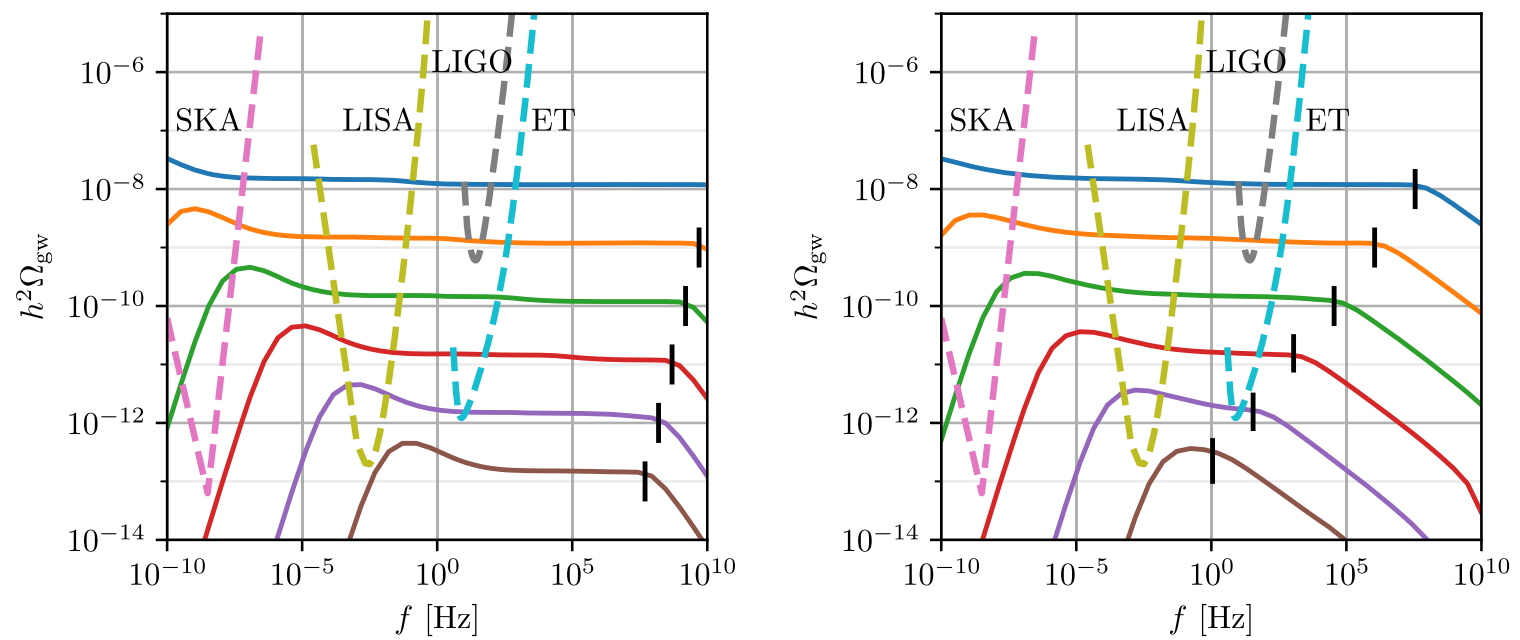

FIG. 3. SBGW including the backreaction of particle emission on the loop distribution. LH panel: kinks on loops, RH panel: cusps on loop. The spectra are cut off at high frequency, as indicated by the black vertical lines. $G \mu$ ranges from $10^{-17}$ (lower curve), through $10^{-15}, 10^{-13}, 10^{-11}, 10^{-9}$, and $10^{-7}$ (upper curve). Also plotted are the power-law integrated sensitivity curves from SKA (pink dashed) [44], LISA (yellow dashed) [45], adv-LIGO (gray dashed) [46], and Einstein Telescope (blue dashed) [47,48].

$\ell_{\mathrm{k}}$ given in Eq. (3) and $P_{j} \propto j^{-5 / 3}$, whereas the right-hand (RH) panel is for cusps with $\ell_{\mathrm{c}}$ given in Eq. (5) and $P_{j} \propto j^{-4 / 3}$. As a result of the nonscaling loop distribution, the spectrum is no longer flat at high frequencies, and, as expected, the effect is more significant for cusps than for kinks since $z_{c}<z_{k}$.

We can estimate the frequency above which the spectrum decays as follows. In the radiation era,

$$
\begin{aligned}
H(z) & =(1+z)^{2} \sqrt{\Omega_{\mathrm{R}}} \mathrm{H}_{0} \\
t(z) & =\frac{1}{2(1+z)^{2}} \frac{1}{\sqrt{\Omega_{\mathrm{R}}} \mathrm{H}_{0}} .
\end{aligned}
$$

At high frequency, the lowest harmonic $j=1$ is expected to dominate [1], so we set $P_{j}=\Gamma \delta_{j, 1}$. Then, using (45) and (46), Eq. (42) simplifies to

$$
\begin{aligned}
\Omega_{\mathrm{gw}}(\ln f) & =2^{4} \frac{16 \pi(\Gamma G \mu)^{2}}{3 \Gamma} \frac{\mathrm{H}_{0}}{f} \Omega_{\mathrm{R}}^{3 / 2} \int_{z_{\mathrm{eq}}}^{z_{\text {friction }}} \mathrm{d} z \mathcal{N}\left(\frac{2}{(1+z) f}, t(z)\right) \\
& \propto \frac{\mathrm{H}_{0}}{f}\left[\int_{z_{\mathrm{eq}}}^{z_{c, k}} \mathrm{~d} z \mathcal{N}\left(\frac{2}{(1+z) f}, t(z)\right)+\int_{z_{c, k}}^{z_{\text {friction }}} \mathrm{d} z \mathcal{N}\left(\frac{2}{(1+z) f}, t(z)\right)\right] . \\
& \simeq \frac{\mathrm{H}_{0}}{f} \int_{z_{\mathrm{eq}}}^{z_{c, k}} \mathrm{~d} z \mathcal{N}\left(\frac{2}{(1+z) f}, t(z)\right) .
\end{aligned}
$$

Here, in going from the second to the third equality, we have used the facts that (i) for $G \mu \gtrsim 10^{-18}$, which is the relevant range for current and future GW detectors, $z_{\text {eq }}<$ $\left(z_{c}, z_{k}\right) \ll z_{\text {friction }}$ [see Eqs. (38), (41), and (44)], and that (ii) the loop distribution above $z_{(c, k)}$ is subdominant; see, e.g., the discussion above Eq. (37) in Sec. III B. Using Eq. (46) as well as the approximation for the loop distribution for $z<z_{k}$ given in Eq. (36), it follows that for kinks

$$
\begin{aligned}
{\left[\Omega_{\mathrm{gw}}(\ln f)\right]_{k} \propto } & \int_{x_{\mathrm{eq}}}^{x_{k}}\left[1+\left(\frac{\ell_{\mathrm{k}} x f^{2}}{8 \mathrm{H}_{0} \sqrt{\Omega_{\mathrm{R}}}}\right)^{2}\right]^{-1 / 2} \\
& \times\left(\gamma_{\mathrm{d}}+x\right)^{-5 / 2} \mathrm{~d} x
\end{aligned}
$$

where we have changed the variable from $z$ to

$$
x=\frac{4}{f}(1+z) \mathrm{H}_{0} \sqrt{\Omega_{\mathrm{R}}}
$$

so that

$$
x_{\mathrm{eq}}=\frac{4}{f}\left(1+z_{\mathrm{eq}}\right) \mathrm{H}_{0} \sqrt{\Omega_{\mathrm{R}}}, \quad x_{k}=\frac{4}{f}\left(1+z_{k}\right) \mathrm{H}_{0} \sqrt{\Omega_{\mathrm{R}}} \text {. }
$$

To understand the frequency dependence of $\Omega_{\mathrm{gw}}$, let us initially focus on the standard NG case, namely, $\ell_{k}=0$. [Here, the same change of variable starting from the first line of Eq. (47) again yields Eq. (48) but with the upper 
bound replaced by $\left.x_{\text {friction }}=4\left(1+z_{\text {friction }}\right) \mathrm{H}_{0} \sqrt{\Omega_{\mathrm{R}}} / f\right]$. Then, Eq. (48) gives

$$
\left[\Omega_{\mathrm{gw}}(\ln f)\right]_{N G} \propto \frac{1}{\left(\frac{f_{\mathrm{eq}}}{f}+1\right)^{3 / 2}}-\frac{1}{\left(\frac{f_{\text {friction }}}{f}+1\right)^{3 / 2}},
$$

where

$$
\begin{aligned}
f_{\text {eq }} & =\frac{4 \mathrm{H}_{0} \sqrt{\Omega_{\mathrm{R}}}\left(1+z_{\mathrm{eq}}\right)}{\gamma_{\mathrm{d}}} \sim \frac{10^{-18}}{G \mu} \mathrm{s}^{-1}, \\
f_{\text {friction }} & =\frac{4 \mathrm{H}_{0} \sqrt{\Omega_{\mathrm{R}}}\left(1+z_{\text {friction }}\right)}{\gamma_{\mathrm{d}}} \sim 10^{10} \mathrm{~s}^{-1},
\end{aligned}
$$

and where in the last equality we have used Eq. (44). At frequencies $f$ for which $f_{\text {friction }} \gg f \gg f_{\text {eq }}$, it follows that $\left[\Omega_{\mathrm{gw}}(\ln f)\right]_{N G} \rightarrow$ constant, meaning that the spectrum is flat, which is the well-known result for NG strings [1].

For $\ell_{\mathrm{k}} \neq 0$, the argument is altered because of the frequency dependence of the term in square brackets in Eq. (48). A further characteristic frequency now enters: this can be obtained by combining the typical scales of the two terms in Eq. (48). Namely, on one hand, from the first term (in square brackets), we have $\ell_{\mathrm{k}} f^{2} \sim 8 \mathrm{H}_{0} \sqrt{\Omega_{\mathrm{R}}} x^{-1}$, and on the other hand, from the second (standard NG) term, we have $x \sim \gamma_{\mathrm{d}}$. Combining these yields the characteristic frequency

$$
f_{k} \sim\left(\frac{8 \mathrm{H}_{0} \sqrt{\Omega_{\mathrm{R}}}}{\ell_{\mathrm{k}} \gamma_{\mathrm{d}}}\right)^{1 / 2}
$$

For $f_{k}>f>f_{\text {eq }}$ the spectrum is still flat, as in the NG case. However, for $f>f_{k}$, it decays since the first term in square brackets in Eq. (48) dominates. With $\ell_{\mathrm{k}}$ given in Eq. (3), $f_{k} \propto(G \mu)^{1 / 4} \beta_{\mathrm{k}}^{-1 / 2}$, and this behavior is clearly shown in Fig. 3, in which $f_{k}$ is shown with a vertical black line for each value of $G \mu$ and we have assumed $\beta_{\mathrm{k}}=1$.

For cusps, the analysis proceeds identically with

$$
f_{c}=\left(\frac{8 \mathrm{H}_{0} \sqrt{\Omega_{\mathrm{R}}}}{\ell_{\mathrm{c}} \gamma_{\mathrm{d}}}\right)^{1 / 2} .
$$

Now, upon using $\ell_{\mathrm{c}}$ defined in Eq. (5), we have $f_{c} \propto$ $(G \mu)^{3 / 4} \beta_{\mathrm{c}}^{-1 / 2}$. The spectrum of SGWB in this case is shown in the RH panel of Fig. 3, in which $f_{c}$ is shown with a vertical black line for each value of $G \mu$ and we have taken $\beta_{\mathrm{c}}=1$.

As the figure shows, with $\beta_{\mathrm{c}}=1$ and in the range of $G \mu$ of interest for GW detectors, the decay of $\Omega_{\mathrm{GW}}$ for $f>f_{c}$ is outside the observational window of the LIGO and LISA (and future ET) detectors. To have $f_{c} \sim f_{\text {LIGO }}$, one would require large values of $\beta_{\mathrm{c}}$, which are not expected.

\section{EMISSION OF PARTICLES}

The loops we consider radiate not only GW but also particles. Indeed, for loops with kinks, from Eq. (2),

$$
\left.\dot{\ell}\right|_{\text {particle }}=-\gamma_{\mathrm{d}} \frac{\ell_{\mathrm{k}}}{\ell} \text {. }
$$

The emitted particles are heavy and in the dark particle physics sector corresponding to the fields that make up the string. We assume that there is some interaction of the dark sector with the standard model sector. Then, the emitted particle radiation will eventually decay, and a significant fraction of the energy $f_{\text {eff }} \sim 1$ will cascade down into $\gamma$ rays. Hence, the string network will be constrained by the diffuse gamma-ray bound measured at $\mathrm{GeV}$ scales by Fermi-Lat [19]. This bound is

$$
\omega_{\mathrm{DGRB}}^{\mathrm{obs}} \lesssim 5.8 \times 10^{-7} \mathrm{eV} \mathrm{cm}^{-3},
$$

where $\omega_{\text {DGRB }}$ is the total electromagnetic energy injected since the Universe became transparent to $\mathrm{GeV} \gamma$ rays at $t_{\gamma} \simeq 10^{15} \mathrm{~s}$; see, e.g., Ref. [25].

The rate per unit volume at which string loops lose energy into particles can be obtained by integrating (51) over the loop distribution $n(\ell, t)=t^{-4} \mathcal{N}(\gamma, t)$, namely,

$\Phi_{\mathrm{H}}(t)=\mu \gamma_{\mathrm{d}} \ell_{\mathrm{k}} \int_{0}^{\alpha t} n(\ell, t) \frac{\mathrm{d} \ell}{\ell}=\mu t^{-3} \gamma_{\mathrm{d}} \gamma_{\mathrm{k}} \int_{0}^{\alpha} \frac{\mathcal{N}\left(\gamma^{\prime}, t\right)}{\gamma^{\prime}} \mathrm{d} \gamma^{\prime}$.

The diffuse gamma-ray background (DGRB) contribution is then given by (see, e.g., Ref. [25])

$$
\begin{aligned}
\omega_{\text {DGRB }}= & f_{\text {eff }} \int_{t_{\gamma}}^{t_{0}} \frac{\Phi_{\mathrm{H}}(t)}{(1+z)^{4}} \mathrm{~d} t \\
= & f_{\text {eff }} \mu \gamma_{\mathrm{d}} \int_{t_{\gamma}}^{t_{0}} \frac{\gamma_{\mathrm{k}}(t)}{t^{3}(1+z(t))^{4}}\left[\int_{0}^{\alpha} \frac{\mathcal{N}\left(\gamma^{\prime}, t\right)}{\gamma^{\prime}} \mathrm{d} \gamma^{\prime}\right] \mathrm{d} t \\
= & \Gamma\left(8.4 \times 10^{39}\right) f_{\text {eff }}\left(\frac{G \mu}{c^{4}}\right)^{2} \\
& \times \int_{t_{\gamma}}^{t_{0}} \frac{\gamma_{\mathrm{k}}(t)}{t^{3}(1+z(t))^{4}}\left[\int_{0}^{\alpha} \frac{\mathcal{N}\left(\gamma^{\prime}, t\right)}{\gamma^{\prime}} \mathrm{d} \gamma^{\prime}\right] \mathrm{d} t \mathrm{eV} \mathrm{cm}^{-3},
\end{aligned}
$$

where in the last line we have explicitly put in factors of $c$ converted to physical units of $\mathrm{eV} / \mathrm{cm}^{3}$. For cusps, one finds

$$
\begin{aligned}
\omega_{\text {DGRB }}= & \Gamma\left(8.4 \times 10^{39}\right) f_{\text {eff }}\left(\frac{G \mu}{c^{4}}\right)^{2} \\
& \times \int_{t_{\gamma}}^{t_{0}} \frac{\sqrt{\gamma_{\mathrm{c}}(t)}}{t^{3}(1+z(t))^{4}}\left[\int_{0}^{\alpha} \frac{\mathcal{N}\left(\gamma^{\prime}, t\right)}{\sqrt{\gamma^{\prime}}} \mathrm{d} \gamma^{\prime}\right] \mathrm{d} t \mathrm{eVcm}^{-3} .
\end{aligned}
$$

In the matter-dominated era, the loop distribution is dominated by those loops produced in the radiation era but 

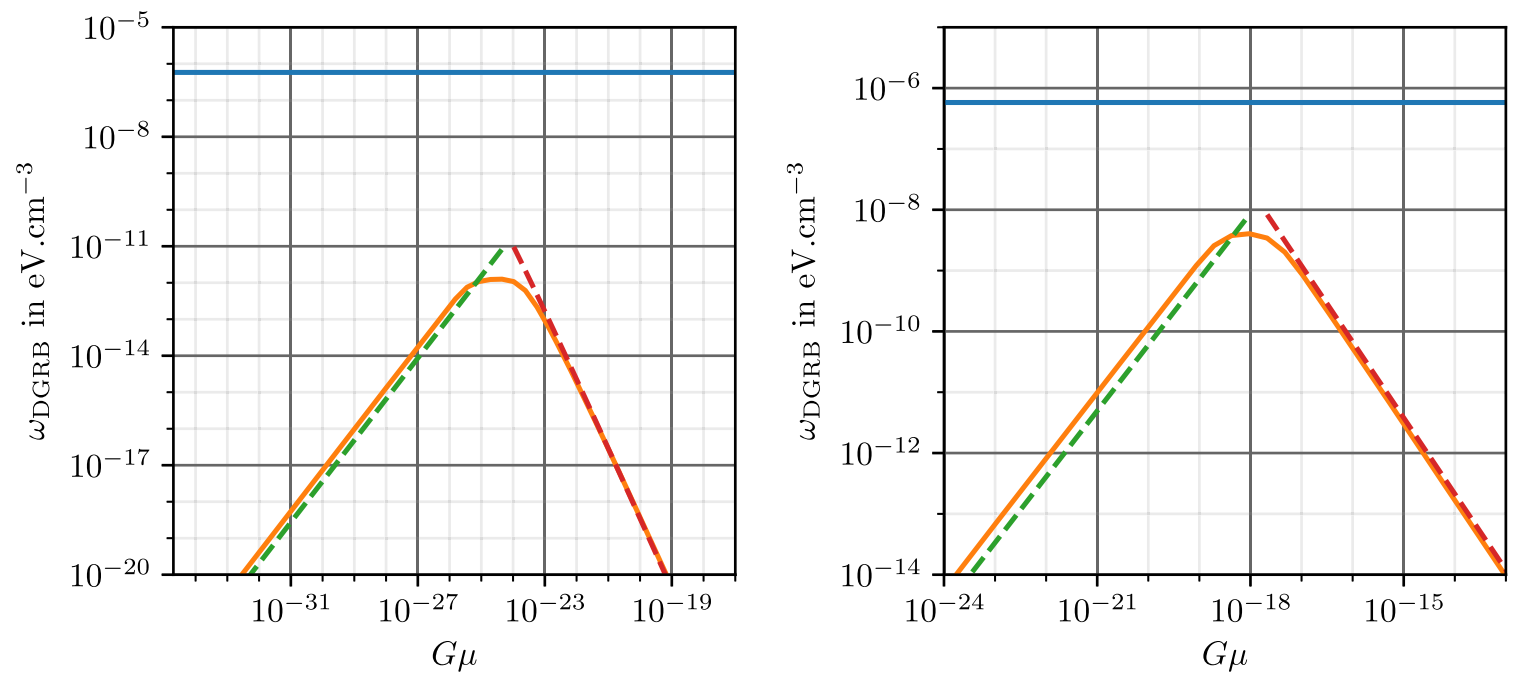

FIG. 4. Contribution of cosmic strings to the diffuse gamma-ray background. The (blue) horizontal line is the experimental constraint from Fermi-LAT, while the (orange) line is the exact numerical calculation for kinks (LH panel) and cusps (RH panel). On either side of the maxima, the slope and amplitude can be estimated using the results of previous sections. Kinks: for low $G \mu$, the slope is $9 / 8$ (dashed green line), and for high $G \mu$, it depends on $\mu^{-2} \log (\mu)$ (dashed red line). Cusps: For low $G \mu$, the slope is 13/12 (dashed green line), and for high $G \mu$, it is $-5 / 4$ (dashed red line). The slightly different amplitude between the numerical calculation and the analytical one is because the latter assumes a matter-dominated Universe, and hence neglects effects of late-time acceleration.

decay in the matter era; its general expression is given in Eq. (23) and can be deduced straightforwardly from the results of Secs. III B and IIIC for kinks and cusps, respectively. We have calculated (54) and (55) numerically, and the results are shown in Fig. 4 for kinks (LH panel) and cusps (RH panel), together with the Fermi-Lat bound. It is clear from this figure that particle radiation from loops containing kinks and/or cusps, with $\ell_{\mathrm{k}}$ and $\ell_{\mathrm{c}}$ given in (3) and (5), are not constrained by the Fermi-Lat data.

The general shape of the spectra in Fig. 4 can be understood from the results of Sec. II. Let us focus on the case of cusps (for kinks, the analysis is similar). First, we can determine the range of $G \mu$ for which the characteristic time $t_{c}$ defined in Eq. (39) falls within the range of integration of (55), namely,

$$
t_{\gamma} \leq t_{c} \leq t_{0} \Leftrightarrow 10^{-19} \lesssim G \mu \lesssim 10^{-18}
$$

[we have assumed $\beta_{\mathrm{c}}=1$, and from Eq. (40), $t=t_{c}$ implies $\left.G \mu \sim 4.6 \times 10^{-18}\left(t_{\mathrm{eq}} / t\right)^{2 / 7}\right]$. This range of $G \mu$ defines the position of the maximum of the DGRB in the RH panel of Fig. 4. For lower $G \mu$, all times in the integration range are smaller than $t_{c}$. As we have discussed in Sec. III C, in this case, the loop distributions are suppressed due to particle radiation; there are fewer loops, and hence fewer particles are emitted, leading to a decrease in the DGRB. This is shown in Fig. 4, and using the results of Sec. III C, one can show that for $G \mu \ll 10^{-19}, \quad \Phi_{\mathrm{H}}(t) \propto \mu^{2 / 3} \ell_{\mathrm{c}}^{-1 / 6}(1+z)^{3} t^{-4 / 3}$, leading to

$$
\omega_{\text {DGRB }} \propto \mu^{2 / 3} \ell_{\mathrm{c}}^{-1 / 6} \propto(G \mu)^{13 / 12} \quad\left(G \mu \ll 10^{-19}\right) .
$$

On the other hand, for $G \mu \gg 10^{-18}$, all times in the integration range are larger than $t_{c}$. There is no suppression of the loop distribution, since GR dominates over particle emission (see Sec. II). But precisely because GR dominates, fewer particles are emitted, and hence we also have a decrease in the DGRB. We now find that $\Phi_{\mathrm{H}}(t) \propto \gamma_{\mathrm{d}}^{-1} \mu \sqrt{\ell_{\mathrm{c}}}(1+z)^{3} t^{-2}$ so that

$$
\omega_{\mathrm{DGRB}} \propto \sqrt{\ell_{\mathrm{c}}} \propto(G \mu)^{-5 / 4},
$$

which is the slope seen in Fig. 4. For kinks, the discussion is very similar, and the slopes are given in the caption of the figure. However, each kink event emits fewer particles, leading to a lower overall DGRB.

\section{CONCLUSION}

Cosmic string loops emit both particle and gravitational radiation. Particle emission is more important for small loops, while gravitational emission dominates for large loops. In this work, we have accounted for both types of radiation in the number density of loops and calculated the expected stochastic gravitational wave background and the diffuse gamma-ray background from strings. Our results show that the number density of loops gets cut off at small lengths due to particle radiation. The strength of the cutoff depends on the detailed particle emission mechanism from strings - if only kinks are prevalent on strings, small loops are suppressed but not as much as in the case when cusps are prevalent (see Fig. 2). The cutoff in loop sizes implies that the stochastic gravitational wave background will get cut off at high frequencies (see Fig. 3). The high-frequency 
cutoff does not affect current gravitational wave detection efforts but may become important for future experiments.

Particle emission from strings can provide an important alternate observational signature in the form of cosmic rays. Assuming that the particles emitted from strings decay into standard model Higgs particles that then eventually cascade into gamma rays, we can calculate the gamma-ray background from strings. This background is below current constraints in the case of both kinks and cusps.

It is important to evaluate more carefully the prevalence of kinks versus cusps on cosmological string loops. In Ref. [11], particle radiation from a loop of a specific shape was studied in which the shape was dictated by general expectations for the behavior of the cosmological string network. That particular loop only contained kinks. It would be of interest to study other loop shapes that are likely to be produced from the network and that contain cusps and to assess if the $1 / \sqrt{\ell}$ dependence in (4) (and assumed throughout this paper) is an accurate characterization of such loops over their lifetimes. In practice, one might expect that if kinks are smoothed out by particle emission, then cusps (perhaps minicusps) must be produced, and if cusps (or minicusps) annihilate, then kinks must be produced. Hence, in reality, the situation might be a combination of both cases. Finally, it would also be interesting to study other loop production functions, particularly those of Refs. [36-38], which predict a larger number of small loops relative to the situation studied in Sec. II B; hence, one might expect a larger gamma-ray background from strings in this case. ${ }^{2}$

\section{ACKNOWLEDGMENTS}

We would like to thank Dimitri Semikoz and Ed Porter for useful discussions and Mark Hindmarsh, Christophe Ringeval, and Géraldine Servant for useful comments and questions on the first draft of this paper. P. A. thanks Nordita for hospitality while this work was in progress. D. A. S. thanks Marc Arène, Simone Mastrogiovanni, and Antoine Petiteau. T. V. thanks APC (Université Paris Diderot) for hospitality through a visiting professorship while this work was being done. T. V. is supported by the U.S. Department of Energy, Office of High Energy Physics, under Award No. DE-SC0019470 at Arizona State University. This work was funded in part by the Gordon and Betty Moore Foundation.

\footnotetext{
${ }^{2}$ Work in progress.
}

[1] A. Vilenkin and E. P.S. Shellard, Cosmic Strings and Other Topological Defects (Cambridge University Press, Cambridge, England, 2000), ISBN 9780521654760.

[2] T. Vachaspati, L. Pogosian, and D. A. Steer, Scholarpedia 10, 31682 (2015).

[3] M. B. Hindmarsh and T. W. B. Kibble, Rep. Prog. Phys. 58, 477 (1995).

[4] B. P. Abbott et al. (LIGO Scientific and Virgo Collaborations), Phys. Rev. D 97, 102002 (2018).

[5] J. J. Blanco-Pillado, K. D. Olum, and X. Siemens, Phys. Lett. B 778, 392 (2018).

[6] P. Auclair et al., arXiv:1909.00819.

[7] G. Vincent, N. D. Antunes, and M. Hindmarsh, Phys. Rev. Lett. 80, 2277 (1998).

[8] M. Hindmarsh, S. Stuckey, and N. Bevis, Phys. Rev. D 79, 123504 (2009).

[9] J. Lizarraga, J. Urrestilla, D. Daverio, M. Hindmarsh, and M. Kunz, J. Cosmol. Astropart. Phys. 10 (2016) 042.

[10] M. Hindmarsh, J. Lizarraga, J. Urrestilla, D. Daverio, and M. Kunz, Phys. Rev. D 96, 023525 (2017).

[11] D. Matsunami, L. Pogosian, A. Saurabh, and T. Vachaspati, Phys. Rev. Lett. 122, 201301 (2019).

[12] T. Vachaspati and A. Vilenkin, Phys. Rev. D 31, 3052 (1985).

[13] C. J. Burden, Phys. Lett. 164B, 277 (1985).

[14] D. Garfinkle and T. Vachaspati, Phys. Rev. D 36, 2229 (1987).
[15] J. J. Blanco-Pillado and K. D. Olum, Phys. Rev. D 96, 104046 (2017).

[16] J. J. Blanco-Pillado and K. D. Olum, Phys. Rev. D 59, 063508 (1999).

[17] K. D. Olum and J. J. Blanco-Pillado, Phys. Rev. D 60, 023503 (1999).

[18] P. Bhattacharjee and G. Sigl, Phys. Rep. 327, 109 (2000).

[19] A. A. Abdo et al. (Fermi-LAT Collaboration), Phys. Rev. Lett. 104, 101101 (2010).

[20] P. Bhattacharjee, Phys. Rev. D 40, 3968 (1989).

[21] J. H. MacGibbon and R. H. Brandenberger, Nucl. Phys. B331, 153 (1990).

[22] J. H. MacGibbon and R. H. Brandenberger, Phys. Rev. D 47, 2283 (1993).

[23] R. H. Brandenberger, A. T. Sornborger, and M. Trodden, Phys. Rev. D 48, 940 (1993).

[24] Y. Cui and D. E. Morrissey, Phys. Rev. D 79, 083532 (2009).

[25] H. F. Santana Mota and M. Hindmarsh, Phys. Rev. D 91, 043001 (2015).

[26] T. Vachaspati, Phys. Rev. D 81, 043531 (2010).

[27] P. Peter and C. Ringeval, J. Cosmol. Astropart. Phys. 05 (2013) 005.

[28] J.-F. Dufaux, J. Cosmol. Astropart. Phys. 09 (2012) 022.

[29] T. Damour and A. Vilenkin, Phys. Rev. Lett. 78, 2288 (1997). 
[30] E. J. Copeland, T. W. B. Kibble, and D. A. Steer, Phys. Rev. D 58, 043508 (1998).

[31] J. J. Blanco-Pillado, K. D. Olum, and B. Shlaer, Phys. Rev. D 83, 083514 (2011).

[32] J. J. Blanco-Pillado, K. D. Olum, and B. Shlaer, Phys. Rev. D 89, 023512 (2014).

[33] C. Ringeval, M. Sakellariadou, and F. Bouchet, J. Cosmol. Astropart. Phys. 02 (2007) 023.

[34] P. Auclair, C. Ringeval, M. Sakellariadou, and D. A. Steer, J. Cosmol. Astropart. Phys. 06 (2019) 015.

[35] L. Lorenz, C. Ringeval, and M. Sakellariadou, J. Cosmol. Astropart. Phys. 10 (2010) 003.

[36] J. Polchinski and J. V. Rocha, Phys. Rev. D 74, 083504 (2006).

[37] J. Polchinski and J. V. Rocha, Phys. Rev. D 75, 123503 (2007).

[38] F. Dubath, J. Polchinski, and J. V. Rocha, Phys. Rev. D 77, 123528 (2008).

[39] N. Aghanim et al. (Planck Collaboration), arXiv:1807.06209.
[40] P. Binetruy, A. Bohé, C. Caprini, and J.-F. Dufaux, J. Cosmol. Astropart. Phys. 06 (2012) 027.

[41] P. A. R. Ade et al. (Planck Collaboration), Astron. Astrophys. 571, A25 (2014).

[42] R. R. Caldwell and B. Allen, Phys. Rev. D 45, 3447 (1992).

[43] P. Binetruy, A. Bohe, T. Hertog, and D. A. Steer, Phys. Rev. D 80, 123510 (2009).

[44] G. Janssen et al., Proc. Sci., AASKA14 (2015) 037 [arXiv: 1501.00127].

[45] C. Caprini, D. G. Figueroa, R. Flauger, G. Nardini, M. Peloso, M. Pieroni, A. Ricciardone, and G. Tasinato, J. Cosmol. Astropart. Phys. 11 (2019) 017.

[46] B. P. Abbott et al. (LIGO Scientific and Virgo Collaborations), Phys. Rev. Lett. 118, 121101 (2017); 119, 029901(E) (2017).

[47] M. Punturo et al., Classical Quantum Gravity 27, 194002 (2010).

[48] S. Hild et al., Classical Quantum Gravity 28, 094013 (2011). 